\title{
IFPA Meeting 2012 Workshop Report III: Trophoblast deportation, gestational trophoblastic disease, placental insufficiency and fetal growth restriction, trophoblast over-invasion and accreta-related pathologies, placental thrombosis and fibrinolysis
}

\author{
A. Al-Khan ${ }^{a}$, J.N. Bulmer ${ }^{b}$, F. Chantraine ${ }^{c}$, C.P. Chen ${ }^{\mathrm{d}}$, Q. Chen ${ }^{\mathrm{e}}$, S. Collins ${ }^{\mathrm{f}}, \mathrm{T}_{\text {. Cotechini }}{ }^{\mathrm{g}}$, J.S. Fitzgerald ${ }^{\mathrm{h}}$,
} M. He ${ }^{\mathrm{i}}$, O. Holland ${ }^{\mathrm{j}}$, T.H. Hung ${ }^{\mathrm{k}}$, N.P. Illsley ${ }^{\mathrm{a}}$, K. Ino ${ }^{1}$, T. Iwaki ${ }^{\mathrm{m}}$, N. Kanayama ${ }^{\mathrm{m}}$, E. Kaneki ${ }^{\mathrm{n}}$, H. Katabuchi $^{\mathrm{o}}$,

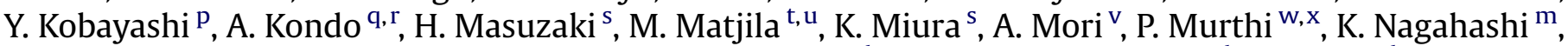

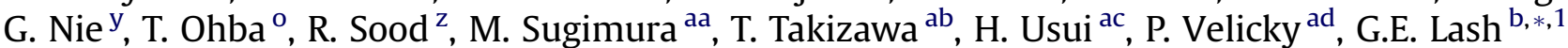

${ }^{a}$ Division of Maternal-Fetal Medicine and Surgery, Department of Obstetrics and Gynaecology, Hackensack University Medical Centre, Hackensack, NJ, USA

${ }^{\mathrm{b}}$ Institute of Cellular Medicine, Newcastle University, Newcastle upon Tyne, UK

'Obstetrics and Gynaecology, CHR Citadell, University of Liege, Liege, Belgium

${ }^{\mathrm{d}}$ Division of High Risk Pregnancy, Mackay Memorial Hospital, Taipei, Taiwan

e Department of Obstetrics and Gynaecology, Wuxi Maternity and Child Health Hospital, Nanjing Medical University, China

${ }^{\mathrm{f}}$ The Nuffield Department of Obstetrics and Gynaecology, University of Oxford, Oxford, UK

${ }^{\mathrm{g}}$ Department of Biomedical and Molecular Sciences, Queen's University, Kingston, Ontario, Canada

${ }^{\mathrm{h}}$ Placenta-Labor, Obstetrics Department, University Hospital Jena, Jena, Germany

${ }^{i}$ Department of Pathology and Laboratory Medicine, Women E Infants Hospital/Warren Alpert Medical School of Brown University, Providence, RI, USA

${ }^{\mathrm{j}}$ Department of Obstetrics and Gynaecology, Faculty of Medical and Health Sciences, The University of Auckland, Auckland, New Zealand

${ }^{\mathrm{k}}$ Department of Obstetrics and Gynaecology, Chang Gung Memorial Hospital, Taipei, Taiwan

${ }^{1}$ Department of Obstetrics and Gynaecology, Wakayama Medical University, Wakayama, Japan

${ }^{\mathrm{m}}$ Department of Obstetrics and Gynaecology, Hamamatsu University School of Medicine, Japan

${ }^{\mathrm{n}}$ Department of Obstetrics and Gynaecology, Graduate School of Medical Science, Kyushu University, Fukuoka, Japan

${ }^{\circ}$ Department of Obstetrics and Gynaecology, Faculty of Life Sciences, Kumamoto University, Kumamoto, Japan

${ }^{\mathrm{p}}$ Department of Obstetrics and Gynaecology, School of Medicine, Keio University, Tokyo, Japan

${ }^{\mathrm{q}}$ Aiiku Maternal and Child Health Center Hospital, Tokyo, Japan

${ }^{\mathrm{r}}$ Tokai University School of Medicine, Kanagawa, Japan

${ }^{s}$ Department of Obstetrics and Gynaecology, Nagasaki University Graduate School of Biomedical Sciences, Nagasaki, Japan

${ }^{\mathrm{t}}$ Department of Obstetrics and Gynaecology, Groote Schuur Hospital, Cape Town, South Africa

${ }^{\mathrm{u}}$ MRC/UCT Receptor Biology Unit, Institute of Infectious Disease and Molecular Medicine, Division of Medical Biochemistry, University of Cape Town, Cape Town, South Africa

${ }^{\mathrm{v}}$ Tokyo City University, Tokyo, Japan

${ }^{\mathrm{w}}$ Department of Perinatal Medicine Pregnancy Research Centre, University of Melbourne, Melbourne, Victoria, Australia

x Department of Obstetrics and Gynaecology, Royal Women's Hospital, Parkville, Victoria, Australia

${ }^{y}$ Prince Henry's Institute of Medical Research, Monash Medical Centre, Melbourne, Victoria, Australia

${ }^{\mathrm{z}}$ Medical College of Wisconsin, Milwaukee, WI, USA

aa Juntendo University, Tokyo, Japan

${ }^{\mathrm{ab}}$ Department of Molecular Medicine and Anatomy, Nippon Medical School, Tokyo, Japan

${ }^{\mathrm{ac}}$ Department of Reproductive Medicine, Graduate School of Medicine, Chiba University, Chiba, Japan

${ }^{\text {ad }}$ Department of Obstetrics and Fetal-Maternal Medicine, Reproductive Biology Unit, Medical University of Vienna, Vienna, Austria

\section{A R T I C L E I N F O}

Article history:

Accepted 22 November 2012

Keywords:

Trophoblast deportation

\section{A B S T R A C T}

Workshops are an important part of the IFPA annual meeting as they allow for discussion of specialized topics. At IFPA meeting 2012 there were twelve themed workshops, five of which are summarized in this report. These workshops related to various aspects of placental biology but collectively covered areas of clinical research and pregnancy disorders: 1) trophoblast deportation; 2) gestational trophoblastic

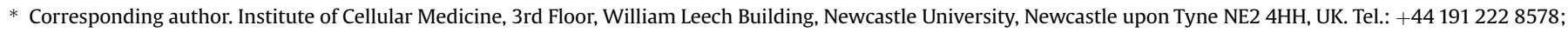
fax: +441912225066.

E-mail addresses: gendie.lash@ncl.ac.uk, g.e.lash@ncl.ac.uk (G.E. Lash).

1 GEL edited this manuscript based on contributions from the other authors. 
Gestational trophoblastic disease Placental insufficiency Fetal growth restriction

Trophoblast over-invasion

Placental thrombosis and fibrinolysis disease; 3) placental insufficiency and fetal growth restriction; 4) trophoblast overinvasion and accretarelated pathologies; 5 ) placental thrombosis and fibrinolysis.

(ㄷ) 2012 Published by IFPA and Elsevier Ltd.

\section{Trophoblast deportation/placental-maternal exchange (pre-natal genetic diagnosis, pre-eclampsia or pregnancy- related diseases)}

Chairs: Hideaki Masuzaki and Chie-Pein Chen.

Speakers: Justine Fitzgerald, Olivia Holland, Kiyonori Miura, Guiying Nie, Toshihiro Takizawa.

\subsection{Outline}

During pregnancy the placenta releases a number of different factors into the maternal circulation; these factors include trophoblast derived material, cell-free DNA/RNA, growth factors and proteases. Altered levels of these different factors have been associated with progression of pregnancy complications such as pre-eclampsia. Indeed, circulating levels of several growth factors have previously been shown to be altered prior to clinical presentation of pre-eclampsia. Increasingly non-invasive means of clinical diagnosis are being sought, and the same holds true for complications of pregnancy. This workshop sought to discuss some of the novel circulating markers currently being examined for diagnosis/ prediction of complications of pregnancy.

\subsection{Summary}

Kiyonori Miura discussed identification of pregnancy-associated microRNAs (miRNAs) and their circulating levels in plasma from normal pregnant women and women with pre-eclampsia. The association between the circulating levels of pregnancy-associated miRNA and pre-eclampsia was investigated. Eighty-two placentapredominantly expressed miRNAs were selected, of which 24 were identified as pregnancy-associated miRNAs. The chromosome 19 miRNA cluster (C19MC) at $19 q 31.42$ is specifically expressed in the placenta (66.7\%). As cell-free pregnancy-related miRNA markers, has-miR-515-3p and has-miR-519b-3p were selected from $3^{\prime}$ and $5^{\prime}$ region of C19MC. The plasma concentration of C19MC-derived miRNAs (has-miR-515-3p and has-miR-519b-3p) increased significantly in plasma from women with pre-eclampsia compared to that from normal pregnancy. Thus, pregnancy-associated miRNAs may be useful molecular markers for monitoring the presence of preeclampsia.

Toshihiro Takizawa discussed dysregulation of placental hydroxysteroid (17-beta) dehydrogenase 1 (HSD17B1) by miR-210 and miR-518c in pre-eclampsia, with plasma levels of HSD17B1 as a novel marker for predicting late-onset pre-eclampsia. It was identified that miR-210 and miR-518c were upregulated in human pre-eclamptic placentas. These miRNAs were shown to target the mRNA encoding HSD17B1, a placenta-specific steroidogenic enzyme, expression of which is significantly decreased in preeclamptic placentas. Furthermore, it was found that plasma HSD17B1 protein levels reflected its placental expression. A prospective cohort study of plasma HSD17B1 revealed that reducing plasma level of HSD17B1 precedes the onset of preeclampsia and is a potential prognostic factor for pre-eclampsia.

Justine Fitzgerald discussed the thrombogeneic potential of syncytiotrophoblast microparticles. Syncytiotrophoblast microparticles (STBMs) are involved in inflammation, immunomodulation and endothelial dysfunction during pre-eclampsia. Recent data indicate that STBMs also participate in coagulation, in particular STBMs derived from pre-eclamptic placentae trigger thrombin generation in a tissue factor-dependent manner. Data was presented confirming the thrombogeneic potential of STBMs: overall oxygen stabilized STBM release, as well as STBM-mediated thrombin activation, fibrin production and maximum platelet aggregation. Insufficient placental oxygen resulted in the opposite; it elevated STBM-shedding, boosted thrombin activation and fibrin production, while accelerating the rate of platelet aggregation. These emerging studies promise to open up a new research area that sheds light on otherwise under-illuminated aspects of preeclampsia.

Guiying Nie discussed HtrA3 as an early marker for preeclampsia. HtrA3 (high temperature requirement A3), a serine protease of the HtrA family, is important for placental development and cancer progression. Recently, HtrA3 was identified as a potential biomarker for early detection of pre-eclampsia. This prompted the generation of a panel of HtrA3-specific monoclonal antibodies and development of an AlphaLISA assay suitable for large scale screening of human serum. The assay detected significantly higher levels of serum HtrA3 at early gestation (13-14 weeks) in pregnant women who subsequently developed pre-eclampsia than controls in a small cohort. Large cohorts of appropriate serum samples will be screened to determine the specificity and sensitivity of the assay in predicting pre-eclampsia.

Olivia Holland discussed the use of whole genome amplification of syncytial nuclear aggregate DNA as a potential for minimally invasive prenatal diagnostics. Syncytial nuclear aggregates (SNAs) are a potential source of fetal/placental DNA for minimally-invasive prenatal diagnostics. It was investigated whether DNA of sufficient quantity and quality for genetic testing was present in SNAs. Thirtysix individual SNAs were collected in vitro and subjected to whole genome amplification. Single SNAs had a mean DNA yield of 135 $( \pm 120) \mathrm{ng} / \mu \mathrm{L}$. Twenty-five SNAs $(69 \%)$ were considered to have sufficient DNA for downstream applications. These results show that a high proportion of SNAs could be used for genetic testing procedures; and suggest that SNAs have potential for use in minimally-invasive prenatal diagnostics.

\subsection{Conclusions}

This workshop highlighted the potential of circulating biomarkers, such as C19MC-derived miRNAs (has-miR-515-3p and has-miR-519b-3p), HSD17B1 and HtrA3, as predictors of preeclampsia. However, large scale cohorts are needed to validate the specificities and sensitivities of these markers. In addition, longitudinal studies are required to determine if these markers are valid predictors of pre-eclampsia prior to clinical presentation of the disease. Moreover, pre-eclampsia increased STBM-shedding that activated thrombin and fibrin production, and platelet aggregation. Placenta-derived SNAs can be a potential source of fetal DNA for prenatal diagnosis by the use of whole genome amplification.

\section{Gestational trophoblastic disease (GTD)}

Chairs: Hidetaka Katabuchi and Kazuhiko Ino.

Speakers: Qi Chen, Eisuke Kaneki, Yusuke Kobayashi, Takashi Ohba, Hirokazu Usui. 


\subsection{Outline}

Gestational trophoblastic disease (GTD) is a spectrum of diseases with abnormal proliferation arising from placental trophoblast which includes 4 main clinicopathological forms; hydatidiform mole, invasive mole, choriocarcinoma and placental site trophoblastic tumor (PSTT). Hydatidiform mole is an abnormal pregnancy with trophoblast hyperplasia and hydropic chorionic villi, and is divided pathologically and genetically into androgenetic complete mole (CM) and triploid partial mole (PM). Approximately $10-20 \%$ of $\mathrm{CM}$ and $<5 \%$ of PM progress to post-molar gestational trophoblast neoplasia (GTN) requiring chemotherapy. Therefore, correct differential diagnosis of $\mathrm{CM}$ and $\mathrm{PM}$ is important for the management of these patients and early detection of GTN. The mechanism by which hydatidiform mole progresses to invasive mole or choriocarcinoma remains unclear. Multiple factors may be involved including up- or down-regulation of specific oncogenes and their proteins, some angiogenic factors and interaction with host immune cells. Although the overall cure rate in treating GTN is currently $>90 \%,-10 \%$ of choriocarcinoma will develop chemoresistance and it remains to be fatal; thus, novel therapeutic strategies are needed to achieve $100 \%$ survival.

This workshop included presentations on recent advances and current research topics in the diagnosis of hydatidiform molar pregnancy as well as in the treatment of post-molar GTN.

\subsection{Summary}

Takashi Ohba discussed the clinical features of histologically confirmed early molar pregnancies. Early detection of pregnancy has resulted in earlier diagnosis of hydatidiform mole. Early stage hydatidiform moles are not hydropic but appear as thickened intrauterine layers with an irregular surface. However, these features are not pathognomonic because similar findings are also observed in non-molar abortion. Evaluation of early hydatidiform mole cannot predict impending post-molar trophoblast disease. Follow-up of hCG levels, similar to the management of classical mole, is also required to detect any subsequent development of post-molar trophoblast disease.

Eisuke Kaneki discussed the incidence of post-molar gestational trophoblast disease in androgenetic moles and the morphological features associated with low risk, post-molar gestational trophoblast disease. The outcomes of 200 genetically diagnosed molar pregnancy cases (178 cases of androgenetic mole, 13 cases of dispermic triploids and nine cases of biparental diploids) were reviewed. Twenty-five cases (14\%) of androgenetic moles developed post-molar GTD, none of the patients with dispermic triploids developed post-molar GTD, none of the patients with androgenetic moles who had hydropic villi less than $2 \mathrm{~mm}$ in their shortest diameter developed post-molar GTD. For patients with dispermic triploids the risk of postmolar GTD is extremely low. The risk of post-molar GTD is also low in patients with androgenetic moles with small hydropic villi.

Qi Chen discussed the placental expression of angiogenic growth factors, VEGF and endoglin, in pregnancies with hydatidiform mole. Hydatidiform mole is an abnormal pregnancy with over-proliferation of the placenta, which causes placental dysfunction. Hydatidiform moles are also associated with development of very early onset pre-eclampsia, which may be due to an imbalance of angiogenic growth factor levels. Levels of VEGF and endoglin in hydatidiform moles were studied, with levels of VEGF but not endoglin being significantly increased in hydatidiform molar placentae in comparison to normal placentae. These data suggest the increased levels of angiogenic factors VEGF in hydatidiform moles may link to the mechanism of developing very early onset pre-eclampsia.

Yusuke Kobayashi discussed the establishment of a new choriocarcinoma cell line, induced choriocarcinoma cell-1 $\left(\mathrm{iC}^{3}-1\right)$, which was generated from HTR8/SVneo retrovirally transduced with activated HRAS. Interestingly, $\mathrm{iC}^{3}-1$ produces lethal tumors consisting of syncytiotrophoblast and cytotrophoblast cells, mimicking the clinical pathology. Microarray analysis between $\mathrm{iC}^{3}$ 1 and parental HTR8/SVneo revealed that $\mathrm{iC}^{3}-1$ expressed matrix metalloproteinase (MMP) and EMT (epithelial-mesenchymal transition) related genes, as well as SOX3. Treatment with shSOX3 attenuated the tumorigenic activity of $\mathrm{iC}^{3}-1$, suggesting SOX3 overexpression might be critically involved in the tumorigenesis of choriocarcinoma. Collectively, these data suggest $\mathrm{iC}^{3}-1$ may have the potential to become a powerful tool for investigation of the choriocarcinoma tumorigenesis and pathogenesis.

Hirokazu Usui discussed the relationship between polymorphisms of folate metabolic enzyme genes and GTD. Methotrexate (MTX) is a key drug for treatment of trophoblastic neoplasia. The target of MTX is the folate metabolic pathway. The C677T polymorphism of methylenetetrahydrofolate reductase (MTHFR) is a candidate gene for predictors of MTX treatment. The C677T polymorphism of both peripheral blood cells and molar tissues of low-risk gestational trophoblastic neoplasia (GTN) cases were investigated. The molar CC genotype of MTHFR C677T correlated with a favorable result after 5-days MTX therapy for low-risk GTN.

\subsection{Conclusions}

In this workshop, recent advances and current topics in the diagnosis/management of hydatidiform molar pregnancy as well as in the treatment of post-molar gestational trophoblastic neoplasia (GTN) were discussed. For the correct diagnosis of hydatidiform moles, cytogenetical analysis and immunohistochemical studies including angiogenic growth factor expression are important in addition to conventional ultrasonographic or pathological analyses. Furthermore, molecular analysis of mechanisms for malignant transformation of GTN or genetic analysis of post-molar GTN may contribute to development of novel therapeutic strategies, and also to improvement of survival rates of GTN patients.

\section{Placental insufficiency and fetal growth restriction}

Chairs: Akira Mori and Padma Murthi.

Speakers: Frederic Chantraine, Tiziana Cotechini, Akane Kondo, Padma Murthi.

\subsection{Outline}

Establishment and growth of the placental vasculature are key determinants of fetal growth and development. Placental insufficiency due to inadequate remodeling of the spiral arteries is a reflection of impaired blood flow to the placenta. It is associated with the maternal syndrome of pre-eclampsia and the fetal syndrome of fetal growth restriction (FGR, also known as intrauterine growth restriction, IUGR). The precise mechanism of FGR in most cases remains elusive, although placental insufficiency appears to be a common feature. Placental examination may be treated with less attention than examination of the fetus or the pregnant uterus. The workshop focused on two themes: 1) imaging placental insufficiency associated with FGR; 2) placental insufficiency associated with placental maldevelopment and function. 


\subsection{Summary}

Frédéric Chantraine discussed the use of magnetic resonance imaging (MRI) for imaging placental insufficiency associated with FGR. FGR is associated with an increased risk of perinatal morbidity and mortality. Possible causes are chromosomal defects, congenital malformations, fetal infections and "last but not least" placental insufficiency. Ultrasound is the first choice and standard imaging method for fetal and placental evaluation in FGR. MRI has proven to be of additional diagnostic value in the assessment of abnormal placental invasion like placenta accreta. Furthermore, recent scientific papers highlight the benefit of "functional" MRI in the assessment of placental insufficiency and FGR.

Akane Kondo discussed the use of ultrasound for imaging placental insufficiency associated with FGR. It is still difficult to predict the incidence of severe FGR before it occurs. The stiffness of the maternal abdominal aorta (AA) has been studied in normal pregnant women and compromised pregnant women (increased peripheral resistance). In the normal group, the Stiffness Index (SI) was increased with the maternal age. In the compromised group, aortic diameter and blood pressure were normal, but SI during the second trimester was increased. The compromised women with higher SI had a significantly higher prevalence of FGR $(P<0.001)$. This study shows that a progressive increase of the $\mathrm{SI}$ is associated with severity of the disease.

Padma Murthi discussed expression of the progesterone receptor (PR) in FGR. The definition of FGR is critical, true FGR being defined as a birth weight less than the 10th centile for gestation, together with evidence of fetal health compromise evaluated by Doppler ultrasound. The majority of FGR cases are due to unknown cause, are termed idiopathic and are frequently associated with placental insufficiency. The presented study was aimed to understand the role of progesterone in feto-placental growth by examining expression patterns of PR. It was shown that PR is expressed in the syncytiotrophoblast and chorionic vessels of the placenta, and its expression is decreased in FGR suggesting that PR may contribute to inadequate feto-placental blood flow in idiopathic FGR.

Tiziana Cotechini discussed whether there was a link between abnormal maternal inflammation and development of FGR or preeclampsia. Abnormal maternal inflammation is often associated with FGR. Using a rat model of LPS-induced FGR, it has been investigated whether abnormal maternal inflammation is causally linked to altered utero-placental hemodynamics leading to impaired fetal growth. Daily administration of low-dose LPS leads to an increased spiral artery resistance index, impaired spiral artery remodeling, elevated mean arteriole pressure, and FGR. These LPSinduced alterations were prevented by a TNF- $\alpha$ inhibitor, thereby establishing a critical role of TNF- $\alpha$ in the pathophysiology of inflammation-induced FGR. These findings suggest that management of inflammation may be a potential strategy in the prevention of FGR.

\subsection{Conclusions}

The term placental insufficiency has long existed to indicate a state of inadequate blood flow through the placenta. The advent of ultrasonography has facilitated the study of the placenta. Moreover, MRI and echo-tracking method has proven to be of additional diagnostic value in the assessment of the placenta associated with FGR. Abnormal maternal inflammation and decreased progesterone receptor expression are causally linked to altered utero-placental as well as feto-placental hemodynamics leading to FGR.

\section{Trophoblast over-invasion and accreta-related pathologies}

Chairs: Nick Illsley and Abdullah Al-Khan.

Speakers: Judith Bulmer, Sally Collins, Tai-Ho Hung, Mushi Matjila, Philipp Velicky.

\subsection{Outline}

Accreta-related pathologies and the trophoblast over-invasion which underlies them are becoming a significant area of clinical concern as rising caesarean section rates increase the incidence of these pathologies. The potentially disastrous clinical outcomes in these cases are driving new clinical practices and new methods of diagnosis. These necessitate an understanding of this problem not only from the point of view of the clinician but also by the basic scientists searching for markers of disease initiation, progression and severity. Research into placenta accreta-related pathologies and trophoblast over-invasion is relatively under-developed. Nevertheless we have the advantage of being able to draw on well-established fields of research such as mechanisms of trophoblast invasion and trophoblast under-invasion in pre-eclampsia to help shape new studies in this field. This workshop was designed with several aims in mind: 1) To introduce placentologists to the clinical problems and initiatives in this field; 2) To draw together and discuss possible mechanisms behind the accreta-related pathologies; 3) To assist in linking the efforts of clinical and basic scientists in the field.

\subsection{Summary}

Tai-Ho Hung introduced the clinical practices and problems facing clinicians dealing with accreta-related pathologies. Accretarelated pathologies have increased in incidence at least 10 -fold, from 1 in 30,000 pregnancies in the 1950s to 1 in 2500 today. Much of this increase is due to the rising incidence of caesarean delivery. However many cases of placenta previa and accreta occur in the absence of caesarean scar or any other manipulation of the uterus, suggesting that placenta previa is at least as important as disruption of the endometrium/decidua. Management of placenta accreta has changed remarkably with advances in diagnostic imaging, the emergence of centers and specialized interdisciplinary teams for patient management. The most obvious favorable change has been anticipatory management. This requires ensuring that caesarean delivery and hysterectomy, if needed, occurs in a controlled setting, with advance preparations for hemorrhagic emergency and remote from labor. Nonetheless questions remain as to the optimal techniques for obtaining hemostasis and the timing of delivery in order to maximize maturity of the fetus while minimizing risk to the mother. Techniques reviewed here included Bakri balloon occlusion, embolization, and hypogastric (aka internal iliac) artery ligation.

Sally Collins discussed new studies in diagnostic ultrasound. Maternal mortality and morbidity are reduced when women with placenta accreta deliver in a tertiary care hospital with a multidisciplinary care team. Current diagnosis rests on subjectively assessing ultrasound and MRI images. The degree of severity and therefore the team required at delivery depends on the 'expert opinion'. With a subjective test, and significant consequences if accreta is missed, there is always a risk of over diagnosis, which has significant maternal and neonatal implications. The development of a novel 3-D power Doppler image analysis tool which provides a quantitative assessment of the size and depth of invasion in cases of placenta accreta was discussed.

Judith Bulmer discussed potential mechanisms of trophoblast over-invasion. Extravillous trophoblast cells (EVT) are naturally 
highly invasive, although their ability to invade an extracellular matrix in vitro declines with increasing gestational age. Many different growth factors and cytokines have been proposed to play roles in regulating EVT invasion, with many of these found to inhibit the process. A recent study of placenta accreta demonstrated areas of accreta were often focal, with 'normal' invasion and spiral artery remodeling occurring in the areas of non-accreta. However, in the areas of accreta (with no decidua) trophoblast giant cell numbers and degree of spiral artery transformation were reduced compared to areas with decidua from the same patient. This suggests a critical role for decidua in regulating trophoblast invasion and terminal differentiation.

Philipp Velicky discussed the role of the Notch-dependent RBPJк transcription factor in trophoblast invasion. The canonical Notchmodulator and transcription factor Recombining Binding Protein$\mathrm{J} \kappa$ (RBPJк) and its co-activators, the Mastermind-like proteins (MAML) 2 and MAML3, were shown to be present in first-trimester human villous and extravillous trophoblast (EVT) cells. MAML2 expression significantly increased during trophoblast invasion. Knock-down of RBPJ $\kappa$ in SGHPL-5 trophoblast-like cells using siRNA mediated gene silencing decreased their invasiveness whereas RBPJ $\kappa$-dependent Notch reporter activity was elevated, suggesting a repressive function of RBPJ $\kappa$ in these cells. On the other hand, outgrowth was increased in human first-trimester placental explants upon silencing RBP $\kappa$, indicating elevated proliferative capacity. Taken together, these data suggest that activation of canonical Notch signaling may represent a mechanism to restrain trophoblast invasiveness.

Mushi Matjila discussed possible roles for Kiss in the pathophysiology of placenta accreta. Kiss 1 and kisspeptins have previously been reported as inhibitors of trophoblast cell invasion. In healthy human pregnancies the expression of Kiss1 (mRNA and protein), Kiss1R (mRNA and protein) and MMP9 (mRNA) were greater in the placenta compared with placental bed and decidua parietalis, while VEGF-A (mRNA and protein) and VEGFR2 (mRNA) were highest in the placental bed. Based on these data several hypotheses/scenarios that could explain the pathophysiology of placenta accreta were posed. (1) There may be markedly decreased Kiss 1 transcript and protein expression by the placenta in accreta. (2) In accreta there could be decreased Kiss1 Receptor (Kiss1R) across the placenta, placental bed and decidua parietalis. (3) In placenta accreta overexpression of matrix metalloproteinases (MMPs), MMP9 in particular, by the placenta could explain overinvasion. Interestingly an inverse relationship between placental Kiss1 and MMP9 expression has been documented in pathological pregnancies. (4) That there possibly exists a balance in angiogenesis between the invading trophoblast and native maternal tissues.

\subsection{Conclusions}

It was clear from the presentations and the discussions that followed that the problem of accreta-related pathologies is attracting a significant amount of attention as a result of the caesarean-driven increase in cases. As a result, new collaborative networks are being set up in North America (Drs. Abdulla Al-Khan and Stacy Zamudio/ Hackensack University Medical Center) and Europe (Dr. Frédéric Chantraine/University of Liege) to examine the problem. Initially it is likely that these networks will be concerned with exploring clinical diagnostic and therapeutic modalities. Nevertheless, it is clear that new factors regulating trophoblast invasion are now receiving attention including kisspeptin and Notch signaling. There is now good evidence to suggest that (abnormal) interaction between extravillous trophoblast and decidual cells may be the key to understanding disease mechanisms. This appears to be the direction being taken by basic science researchers investigating this pathology.

\section{Thrombosis and fibrinolysis in the placenta and in still birth}

Chairs: Naohiro Kanayama, Mai He.

Speakers: Takayuki Iwaki, Mai He, Kotomi Nagahashi, Rashmi Sood, Motoi Sugimura.

\subsection{Outline}

Placental circulation is critical for normal fetal growth and maternal health. Thrombosis in the intervillous space can lead to fetal growth restriction, fetal distress and still birth, while fibrinolysis in the placenta can lead to abortion or placental abruption. The balance between coagulation and fibrinolytic factors in the placenta is essential for maintaining pregnancy. This workshop aimed to discuss current issues regarding thrombosis and fibrinolysis in the placenta.

\subsection{Summary}

Kotomi Nagahashi discussed the role of the decidual fibrin layer in the maintenance of pregnancy. Plasminogen activator inhibitor type 1 (PAI-1) is essential for maintenance of pregnancy and its deficiency causes genital bleeding or placental hematoma. This suggests that PAI-1 is closely related to formation of the fibrinoid layer (Nitabuch's layer). PAI-1 protects the Nitabuch's layer from fibrinolysis at the placental attachment site. An increase in fibrinolysis could cause some disorders with genital bleeding in pregnancy.

Takayuki Iwaki discussed the role of blood coagulation and fibrinolytic factors in the placenta. The potential importance of the coagulation, anticoagulation, and fibrinolytic systems in placenta and maintaining pregnancy is based on the observation that fibrin deposits and fibrin degradation products are resident in the fetomaternal surfaces, and lack of maternal fibrinogen results in spontaneous abortion in early pregnancy in humans. Recently, a complete SERPINE1 (PAI-1) deficiency was reported in humans. A pregnancy without supplementation of PAI-1 via fresh frozen plasma resulted in spontaneous abortion.

Rashmi Sood discussed the role of a maternal platelet response to thrombin in regulating placental development and fetal growth in a murine model of thrombophilia-associated pregnancy loss. Interventions aimed at limiting placental thrombosis, such as treatment with low molecular weight heparin (LMWH), are being tested to prevent placental dysfunction and the related sequelae in at-risk pregnancies. The risk-to-benefit balance of antithrombotic prophylaxis during pregnancy is a subject of intense ongoing debate. Results from studies in murine models of placental failure and fetal loss associated with defects in the thrombomodulinProtein $C$ anticoagulation pathway were discussed.

Motoi Sugimura discussed heparin/heparin sulfate (HS)/ CD44v3-mediated signaling in the structural repair of a trophoblast cell layer damaged by mechanical scratching. The effect of heparin/ HS/CD44v3-mediated processes during scratch wound closure was evaluated in monolayer culture of immortalized human trophoblast cells derived from term placenta (TCL-1). The results suggest that heparin/HS/CD44v3-mediated signaling enhances the repair of the trophoblast cells layer damaged with mechanical scratching.

Mai He discussed a clinical case emphasizing the balance of thrombosis and fibrinolysis needed for successful pregnancy. The patient had recurrent first trimester pregnancy loss up to 8 times. Most clinical workup results were negative, except for homozygous 
MTHFR C677T mutation and positive family history. She was given low molecular weight heparin (LMWH) since pregnancy \#7, and clinical decision was made to continue LMWH throughout the last pregnancy (\#9), which led to a live birth at 32 weeks. Placental examination revealed massive perivillous fibrin/fibrinoid deposition and intraparenchymal hematoma, a reflection of dynamic and heterogeneous balance between thrombosis and fibrynolysis. The specific histopathology in the placenta and prior POCs, its impact on pregnancy, and potential mechanisms of heparin were discussed.

\subsection{Conclusions}

The aim of this workshop was to discuss the importance of the blood clotting and fibrinolytic systems in the maintenance of pregnancy. The role of fibrin in decidua was discussed. It was demonstrated that the lack of a fibrinoid layer in decidua leads to abortion or placental abruption. There have been a few previous studies on the relationship between the fibrinoid layer of decidua and maintenance of pregnancy. Although many investigators have focussed on blood coagulation and fibrinolysis of the intervillous space, the data presented here highlighted the importance of decidual fibrinoid in the maintenance of pregnancy. The other presentations focussed on the effect of LMWH on thrombophila, the treatment for hypercoagulative state of intervillous space and decidual vessels. It was agreed that heparin/LMWH treatment is effective for recurrent miscarriage resulting from a hypercoagulative status. It was emphasized that long term heparin/ LMWH treatment is required for successful maintenance of pregnancy in these cases. It is hoped that this workshop has highlighted the importance of research on blood coagulation and fibrinolysis in the placenta as these processes are critical for prevention of pregnancy loss and still birth.

\section{Conflict of interest statement}

None of the authors have any conflict of interest to declare. 